\title{
Nitric oxide mediates hypoxia-induced changes in paracellular permeability of cerebral microvasculature
}

\author{
Karen S. Mark, Amanda R. Burroughs, Rachel C. Brown, Jason D. Huber, and Thomas P. Davis \\ Department of Pharmacology, University of Arizona, Tucson, Arizona 85724-5050
}

Submitted 9 August 2002; accepted in final form 2 September 2003

\begin{abstract}
Mark, Karen S., Amanda R. Burroughs, Rachel C. Brown, Jason D. Huber, and Thomas P. Davis. Nitric oxide mediates hypoxia-induced changes in paracellular permeability of cerebral microvasculature. Am J Physiol Heart Circ Physiol 286: H174-H180, 2004. First published September 4, 2003; 10.1152/ajpheart.00669. 2002.- Ischemic stroke from a reduction in blood flow to the brain microvasculature results in a subsequent decreased delivery of oxygen (i.e., hypoxia) and vital nutrients to endothelial, neuronal, and glial cells. Hypoxia associated with stroke has been shown to increase paracellular permeability of the blood-brain barrier, leading to the release of cellular mediators and brain tissue injury. Whereas reperfusion does not occur in all ischemic strokes, increased permeability has been seen in posthypoxic reoxygenation. Currently, it is unknown whether these deleterious effects result from cellular mechanisms stimulated by decreased oxygen during stroke or posthypoxic reoxygenation stress. This study used primary bovine brain microvessel endothelial cells (BBMECs) to examine the involvement of nitric oxide (NO) as a mediator in hypoxia-induced permeability changes. Hypoxia-induced increased transport of $\left[{ }^{14} \mathrm{C}\right]$ sucrose across BBMEC monolayers compared with normoxia was attenuated by either posthypoxic reoxygenation or inhibition of NO synthase (NOS). The hypoxia-induced permeability effect was further reduced when NOS inhibition was combined with posthypoxic reoxygenation. Additionally, a significant increase in total NO was seen in BBMECs after hypoxic exposure. This correlation was supported by the increased $\left[{ }^{14} \mathrm{C}\right]$ sucrose permeability observed when BBMECs were exposed to the NO donor diethylenetriaamine NONOate. Western blot analyses of NOS isoforms showed a significant increase in the inducible isoform after hypoxic exposure with a subsequent reduction in expression on reoxygenation. Results from this study suggest that hypoxia-induced blood-brain barrier breakdown can be diminished by inhibition of NO synthesis, decreased concentration of NO metabolites, and/or reoxygenation.
\end{abstract}

nitric oxide synthase; blood-brain barrier; endothelial; reoxygenation

THE ENDOTHELIAL CELLS of the cerebral microvasculature form the blood-brain barrier (BBB), which protects the sensitive neuronal and glial cells from peripheral circulating mediators and toxic substances. Whereas it is well known that hypoxic stress leads to ion imbalance, ATP depletion, neurotransmitter release, and neuronal cell death, controversy exists as to whether the tissue injury is due to hypoxic stress itself or posthypoxic reoxygenation $(1,13,28,30,45)$. The response of brain microvessel endothelial cells to hypoxic insult is currently not well understood. Previously, we demonstrated that increased paracellular permeability in brain microvessel endothelial cells following exposure to hypoxic stress was attenuated on reoxygenation (30). Whereas these changes were associated with alterations in tight junctional protein expres-

Address for reprint requests and other correspondence: T. P. Davis, Univ. of Arizona, 1501 N. Campbell, PO 245050, Tucson, AZ 85724-5050 (E-mail: davistp@u.arizona.edu). sion and localization, the underlying intracellular mechanisms that produce this increased permeability remain relatively undefined.

Whereas increased paracellular permeability has been reported following hypoxia $(1,9,14,30)$ and posthypoxic reoxygenation $(43,48)$, cellular mechanisms leading to changes in permeability induced by ischemia-reperfusion are under investigation. The formation of nitric oxide (NO) has been implicated in tissue damage following sustained hypoxia such as that seen in ischemic stroke or high-altitude exposure $(6,38,46)$. Additionally, NO has been associated with increased paracellular permeability of cerebral microvasculature during hypertension (32). Whereas NO has been implicated in hypoxia-induced BBB permeability changes (13) and in posthypoxic reoxygenation alterations $(22,31,45)$, it is unclear whether hypoxic stress or posthypoxic reoxygenation produces fluctuations in NO and ultimately alterations in the BBB. Furthermore, it is important to understand how NO signaling is involved in BBB permeability changes during hypoxia and posthypoxic reoxygenation to treat or prevent brain tissue damage that occurs in ischemic stroke and/or high-altitude exposure.

NO is primarily synthesized from oxygen and arginine by NO synthase (NOS). Studies using knockout mice have shown, in the middle cerebral artery occlusion model, that NO formation from either the neuronal (nNOS) or inducible (iNOS) isoform of NOS is detrimental to cerebral tissue, whereas NO from the endothelial (eNOS) isoform is generally beneficial to cerebral tissue $(20,41)$. Whereas NOS isoforms are different based on tissue and cellular location (i.e., membrane bound or cytoplasmic) and necessary cofactors, modulation of NOS isoform synthesis or activity results in altered NO formation. NO has a variable half-life, of which its breakdown is dependent on several factors, including its inherent concentration, oxygen levels heme proteins, reactive oxygen species (ROS), and the tissue microenvironment. Changes in NO levels or NOS isoforms may induce secondary signaling pathways or morphological changes that underlie the observed increase in paracellular permeability.

Previously, we (30) showed increased paracellular permeability following hypoxic exposure that is partially attenuated on reoxygenation. The present study turns to examining changes in permeability with NOS inhibition, alterations in NOS isoform expression, and total NO levels occurring in the cerebral microvessel endothelial cells under hypoxic stress and posthypoxic reoxygenation. Data presented here support the hypothesis that BBB disruptions induced by hypoxic stress are in part dependent on changes in NO production and/or degra-

The costs of publication of this article were defrayed in part by the payment of page charges. The article must therefore be hereby marked "advertisement" in accordance with 18 U.S.C. Section 1734 solely to indicate this fact. 
dation. Furthermore, recovery of BBB function following posthypoxic reoxygenation and/or NOS inhibition indicates a possible therapeutic approach in the treatment of ischemic stroke and/or high-altitude exposure.

\section{MATERIALS AND METHODS}

Materials. Transwell polyester membrane inserts, minimal essential medium (MEM), and Ham's F-12 media were purchased from Fisher Scientific (St. Louis, MO). $N^{\omega}$-nitro-L-arginine methyl ester (L-NAME), bovine fibronectin, bovine serum albumin, and equine serum were purchased from Sigma Chemical (St. Louis, MO). N-[3(aminomethyl)benzyl]acetamidine (1400W) was purchased from Cayman Chemicals (Ann Arbor, MI). Rat tail collagen (type I) was purchased from Collaborative Biomedical Products (Medford, MA). Bicinchoninic acid (BCA) protein assay kit was purchased from Pierce (Indianapolis, IN). Radioactive $\left[{ }^{14} \mathrm{C}\right]$ sucrose $(462 \mathrm{mCi} / \mathrm{mol}$ specific activity) was purchased from ICN (Irvine, CA). Tris-glycine gels (4-12\%) were purchased from Invitrogen (Carlsbad, CA). All other nutrients, salts, and antibiotics used in the culture media or assay buffers were of cell culture quality from Sigma Chemical.

Antibodies. Rabbit polyclonal eNOS (135 kDa) and iNOS (130 $\mathrm{kDa}$ ) antibodies documented to react with most mammalian species were purchased from Cayman Chemicals (Ann Arbor, MI). AntieNOS is directed against amino acids 1186-1203 of human eNOS and shows no cross-reactivity with iNOS. Similarly, anti-iNOS, directed against NOS from activated macrophages, shows no cross-reactivity with eNOS. Anti-rabbit (IgG) horseradish peroxidase-conjugated secondary antibodies were purchased from Amersham Life Science Products (Arlington Heights, IL).

Isolation and culturing of bovine brain microvessel endothelial cells. Fresh bovine brains were obtained from the University of Arizona Animal Services. Primary bovine brain microvessel endothelial cells (BBMECs), used as an in vitro BBB model, were collected from gray matter of bovine cerebral cortices by using a combination of enzymatic digestion and centrifugal separation methods as previously described (33). BBMECs were seeded $\left(50,000 \mathrm{cells} / \mathrm{cm}^{2}\right)$ on collagen-coated, fibronectin-treated $75-\mathrm{cm}^{2}$ tissue culture flasks or 12-well Transwell polyester membrane inserts $(0.4 \mu \mathrm{m}$ pore $/ 12 \mathrm{~mm}$ diameter). Culture medium consisted of the following: $45 \% \mathrm{MEM}$, 45\% Ham's F-12 nutrient mix, 10 mM HEPES, 13 mM NaHCO 3,50 $\mu \mathrm{g} / \mathrm{ml}$ gentamicin, $10 \%$ equine serum, $2.5 \mu \mathrm{g} / \mathrm{ml}$ amphotericin $\mathrm{B}$, and $100 \mu \mathrm{g} / \mathrm{ml}$ heparin. Cell cultures were grown in a humidified $37^{\circ} \mathrm{C}$ incubator with room air-5\% $\mathrm{CO}_{2}$, and culture medium was replaced every other day until the BBMEC monolayers reached confluency ( 12-14 days).

Hypoxia and reoxygenation treatment. Confluent BBMEC monolayers were subjected to normoxic, hypoxic, or posthypoxic reoxygenation conditions as previously described (30). Briefly, hypoxic BBMEC monolayers were exposed to reduced oxygen $\left(1 \% \mathrm{O}_{2}\right)$ for $24 \mathrm{~h}$ at $37^{\circ} \mathrm{C}$ by using a humidified hypoxic workstation (Coy Laboratories; Grass Lake, MI). After $24 \mathrm{~h}$ of hypoxia treatment, the reoxygenation treatment group was returned to normoxic conditions at $37^{\circ} \mathrm{C}$ for $2 \mathrm{~h}$. BBMEC monolayers exposed to hypoxia were used to assess paracellular permeability, total NO production, and protein expression (measured using Western blot analyses). Changes in BBMEC monolayers following these treatment protocols were compared with control monolayers incubated under normoxic conditions (room air-5\% $\mathrm{CO}_{2}$ ) for $24 \mathrm{~h}$ at $37^{\circ} \mathrm{C}$.

Treatment with NOS inhibitors. To investigate the involvement of NO following hypoxia or posthypoxic reoxygenation-induced effects, L-NAME was used to nonselectively inhibit NOS isoforms, and $1400 \mathrm{~W}$ was used to selectively inhibit iNOS. L-NAME and 1400W were dissolved in PBS before being diluted to a final concentration of $100 \mathrm{nM}$ in culture medium. BBMECs were treated with L-NAME or 1400W $30 \mathrm{~min}$ before and throughout the exposure period (i.e., normoxic, hypoxic, and posthypoxic reoxygenation).
Treatment with NO donors. To investigate whether NO directly effects increased BBB permeability, BBMECs monolayers were treated with $S$-nitroso- $N$-acetyl-DL-penicillamine (SNAP, $30 \mu \mathrm{M}$; $t_{1 / 2}=6 \mathrm{~h}$ ) or diethylenetriaamine NONOate (DETA-NONOate, 10 or $100 \mu \mathrm{M} ; t_{1 / 2}=20 \mathrm{~h}$ ) to assess permeability changes. Both compounds were purchased from Cayman Chemicals and prepared according to specifications, with SNAP being dissolved in $1 \mathrm{~N} \mathrm{NaOH}$ to make a stock solution of $3 \mathrm{mM}$, and DETA-NONOate was dissolved in deoxygenated PBS to make a stock solution of $1 \mathrm{mM}$ before being diluted with culture medium for the experimental concentrations. BBMEC monolayers treated with $\mathrm{NO}$ donors were incubated under normoxic conditions for 6 or $24 \mathrm{~h}$ at $37^{\circ} \mathrm{C}$ and compared with control monolayers (culture medium alone).

Gas analysis of cell culture medium. $\mathrm{Po}_{2}$ (in $\mathrm{mmHg}$ ) was measured in the cell culture medium after exposure of BBMEC monolayers to normoxic (control), hypoxic, or posthypoxic reoxygenation conditions, with and without NOS inhibitor treatment, using an ABL505 (Radiometer; Copenhagen, Denmark).

$B B M E C$ monolayer permeability experiments. After exposure to treatment conditions, i.e., NOS inhibitors, NO donors, normoxic, hypoxic, or posthypoxic reoxygenation conditions, confluent BBMEC monolayers were incubated with assay buffer consisting of (in $\mathrm{mM}$ ) $122 \mathrm{NaCl}, 3 \mathrm{KCl}, 1.4 \mathrm{CaCl}_{2}, 1.2 \mathrm{MgSO}_{4}, 25 \mathrm{NaHCO}_{3}, 10 \mathrm{HEPES}, 10$ glucose, and $0.4 \mathrm{~K}_{2} \mathrm{HPO}_{4}$ for $30 \mathrm{~min}$ at $37^{\circ} \mathrm{C}$ under the same oxygen conditions. Paracellular permeability across BBMEC monolayers was determined by measuring the transport of the membrane-impermeant marker $\left[{ }^{14} \mathrm{C}\right]$ sucrose. Briefly, $\left[{ }^{14} \mathrm{C}\right]$ sucrose $(0.5 \mu \mathrm{Ci})$ was added to the luminal side (upper compartment of the Transwell), and samples (50 $\mu l$ ) were removed from the abluminal side (lower chamber of the Transwell) at $0,30,60$, and $120 \mathrm{~min}$. The volume withdrawn was replaced with fresh assay buffer. The concentration of $\left[{ }^{14} \mathrm{C}\right]$ sucrose applied to the luminal side (donor chamber) was determined by removing samples $(50 \mu \mathrm{l})$ at time 0 . The amount of radioactivity was determined by using a Beckman LS5000 TD beta-counter. Permeability coefficients (PC) for $\left[{ }^{14} \mathrm{C}\right]$ sucrose were expressed as previously described (11) where $\mathrm{V}$ is the volume in the receiver chamber (1.5 $\left.\mathrm{cm}^{3}\right)$, SA is the surface area of the cell monolayer $\left(1 \mathrm{~cm}^{2}\right), C_{d}$ is the concentration of marker in the donor chamber at time 0 , and $\mathrm{C}_{\mathrm{r}}$ is the concentration of marker in the receiver chamber at time $T$

$$
\mathrm{PC}(\mathrm{cm} / \mathrm{min})=\frac{\mathrm{V}}{\mathrm{SA} \cdot \mathrm{C}_{\mathrm{d}}} \cdot \frac{\mathrm{C}_{\mathrm{r}}}{T}
$$

Enzyme immunoassay for total NO. Total NO release was determined by measuring the accumulation of $\mathrm{NO}$, nitrite $\left(\mathrm{NO}_{2}^{-}\right)$, and nitrate $\left(\mathrm{NO}_{3}^{-}\right)$in the extracellular medium by the Greiss method by using a total NO assay kit (R\&D Systems; Minneapolis, MN). After treatment of BBMECs with L-NAME or culture medium alone during exposure to normoxia, hypoxia, or posthypoxic reoxygenation, extracellular medium was removed (under treatment conditions) for analysis. $\mathrm{NO}$ and $\mathrm{NO}_{3}^{-}$were converted to $\mathrm{NO}_{2}^{-}$by mixing samples with $\mathrm{NADPH}$ and nitrate reductase and incubating at $37^{\circ} \mathrm{C}$ for $30 \mathrm{~min}$. An equal volume of Greiss reagent [sulfanilamide and $N$-(1-napthyl)ethylenediamine in $2 \mathrm{~N} \mathrm{HCl}$ ] was added to each sample and incubated at room temperature for $10 \mathrm{~min}$ to produce an azo-dye product. The optical density of each sample was read spectrophotometrically at 540 $\mathrm{nm}$ against a $\mathrm{NaNO}_{2}$ standard curve with a detection limit of $3.12 \mu \mathrm{M}$. Results were expressed as micromoles per milligram of protein to normalize for cell number, where the cellular protein was determined by using the BCA assay. Total NO was also measured in the culture medium alone and with L-NAME $(100 \mathrm{nM})$ under normoxic and hypoxic conditions to assess the contribution of NO from media without cells.

Western blot protein analysis. After exposure to normoxia, hypoxia, or posthypoxic reoxygenation alone and in combination with L-NAME treatment, protein was isolated from treated BBMEC monolayers using the TRI reagent protocol (Sigma). Briefly, protein was 
separated from RNA and DNA by chloroform and ethanol extraction and then precipitated by using isopropanol followed by washes with guanidinium HCl-95\% ethanol before dissolution in 1\% SDS. Protein was quantified by using the BCA protein assay.

Protein samples $(20 \mu \mathrm{g})$ were separated by using an electrophoretic field on Novex 4-12\% Tris-glycine gels at $125 \mathrm{~V}$ for $75-90 \mathrm{~min}$. The proteins were transferred to polyvinylidene fluoride membranes with $240 \mathrm{~mA}$ at $4^{\circ} \mathrm{C}$ for $30 \mathrm{~min}$. Membranes were blocked using 5\% nonfat milk-Tris-buffered saline (TBS: $20 \mathrm{mM}$ Tris base and $137 \mathrm{mM} \mathrm{NaCl}$, $\mathrm{pH}$ 7.6) with $0.1 \%$ Tween 20 and were incubated overnight at $4{ }^{\circ} \mathrm{C}$ with primary antibodies (1:1,000-1:2,000 dilution) in PBS- $0.5 \%$ BSA. The membranes were washed with $5 \%$ nonfat milk-TBS buffer before incubation with the appropriate secondary antibody (in PBS$0.5 \% \mathrm{BSA}$ ) at a 1:2,000 dilution for $30 \mathrm{~min}$ at room temperature. Membranes were developed by using the enhanced chemiluminescence method (ECL; Amersham), and protein bands were visualized on X-ray film (Kodak; Rochester, NY). Scion Image software (courtesy of National Institutes of Health) was used for semiquantitative analysis of protein expression, and the results were normalized to the amount of protein loaded.

Data analysis. Statistical analyses of the data were done using one-way and two-way analysis of variance with Tukey's multirange post hoc comparison of the means (SigmaStat; SPSS).

\section{RESULTS}

Gas analysis of treated BBMECs. $\mathrm{Po}_{2}$ (in $\mathrm{mmHg}$ ) levels were measured in the culture medium of BBMEC monolayers subjected to hypoxic and posthypoxic reoxygenation conditions in the presence and absence of NOS inhibitor and were compared with normoxic controls. Within $10 \mathrm{~min}$ of hypoxic exposure, culture medium $\mathrm{PO}_{2}$ levels dropped significantly (35-52 $\mathrm{mmHg}$ ) and remained level throughout the exposure period with a rapid return in $\mathrm{Po}_{2}$ to control levels (139-156 $\mathrm{mmHg}$ ) on reoxygenation. There was no difference in $\mathrm{Po}_{2}$ levels of the culture medium with the NOS inhibitor compared with the culture medium alone.

Paracellular permeability of $\left[{ }^{14} C\right]$ sucrose in BBMECs. There was a significant effect of oxygen concentration on the BBMEC monolayer permeability $\left[F_{2,116}=77.14 ; P<0.001\right]$. An increase in $\left[{ }^{14} \mathrm{C}\right]$ sucrose permeability was observed in BBMEC monolayers exposed to hypoxia compared with normoxia (11.10 vs. $4.79 \mathrm{~cm} / \mathrm{min} \times 10^{-4}$, respectively; Fig. 1). Reoxygenation showed an attenuating effect (51\%) compared with the hypoxia-induced increased permeability (7.87 vs. $11.10 \mathrm{~cm} / \mathrm{min} \times 10^{-4}$, respectively). Inhibition of NO synthesis by L-NAME, a nonselective NOS inhibitor, or $1400 \mathrm{~W}$, an iNOS-selective inhibitor, had a significant attenuating effect on the hypoxia-induced permeability changes. This is apparent with the $50 \%$ reduction in permeability of L-NAME-treated monolayers and the $36 \%$ reduction in $1400 \mathrm{~W}$-treated monolayers compared with controls after hypoxic exposure (8.28 and 8.85 compared with $11.10 \mathrm{~cm} / \mathrm{min} \times 10^{-4}$, respectively; Fig. 1). Furthermore, BBMEC monolayers treated with LNAME or $1400 \mathrm{~W}$ showed a synergistic reduction $(73 \%$ and $79 \%$, respectively) in hypoxia-induced permeability effects when combined with $2 \mathrm{~h}$ posthypoxic reoxygenation (6.52 or 6.17 compared with $11.10 \mathrm{~cm} / \mathrm{min} \times 10^{-4}$, respectively). As previously reported, these permeability changes are not due to changes in cell viability (30).

Release of total NO from BBMECs. Changes in total NO released from confluent BBMEC monolayers during hypoxic and posthypoxic reoxygenation exposure were compared with NO released under normoxic conditions. Whereas a low con-

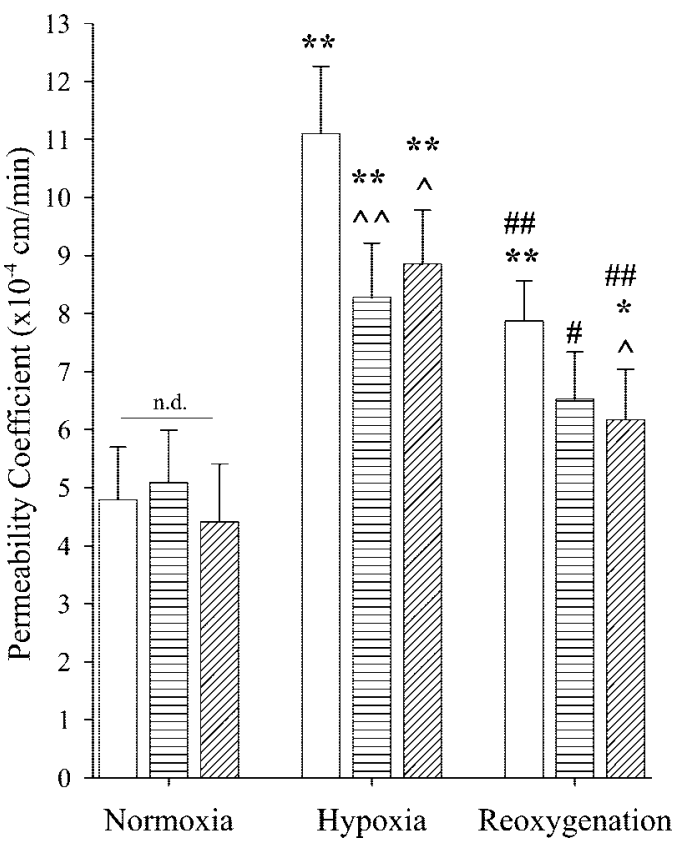

Fig. 1. Permeability coefficient of bovine brain microvessel endothelial cells (BBMECs) after exposure to normoxic $(24 \mathrm{~h})$, hypoxic $(24 \mathrm{~h})$, or posthypoxic reoxygenation $(2 \mathrm{~h})$ conditions. Open bars, monolayers in culture medium alone (control); striped bars, monolayers treated with a nonselective nitric oxide (NO) synthase (NOS) inhibitor $N^{\omega}$-nitro-L-arginine methyl ester (LNAME, $100 \mathrm{nM}$ ); hatched bars, monolayers treated with a selective inhibitory NOS (iNOS) inhibitor (1400W, $100 \mathrm{nM}$ ). Results are means \pm SE with $n=$ 15. Data were analyzed using two-way ANOVA with Tukey's post hoc comparison: $* P<0.05, * * P<0.001$ compared with normoxic condition; $\# P<0.05, \# \# P<0.001$ compared with hypoxic condition; ${ }^{\wedge} P<0.05,{ }^{\wedge} \wedge P<$ 0.001 compared with treatment control. $F_{2,116}=77.14$ and $P<0.001$ for differences based on changes in oxygen, $F_{2,116}=8.938$ and $P<0.001$ for differences based on NOS inhibition, and $F_{4,108}=3.002$ with $P<0.05$ for interaction between oxygen level and inhibitor. n.d., Not determined.

centration of NO $(<20 \%)$ was detected in the culture medium itself (i.e., no BBMECs), there was no difference between the culture medium alone or with L-NAME. Furthermore, there was no difference between media exposed to normoxic or hypoxic conditions (data not shown). In contrast, a significant increase in total NO released from BBMECs was observed after hypoxic exposure $(P<0.001$; Table 1$)$. Posthypoxic reoxygenation resulted in a $58 \%$ reduction in total NO levels compared with BBMEC monolayers under hypoxic conditions. There was also a significant reduction $(50 \%, P<0.001)$ in total NO levels from BBMECs pretreated with L-NAME compared with the monolayers in the culture medium alone during hypoxic exposure. Although this NOS inhibitor had an attenuating effect in hypoxia-induced NO levels, L-NAME itself had a stimulating effect on NO release with basal levels increasing $47 \%$ compared with normoxic controls (Table 1).

Permeability in NO donor-treated BBMEC monolayers. There was a significant effect of both NO donor and time of exposure in $\left[{ }^{14} \mathrm{C}\right]$ sucrose permeability across treated BBMEC monolayers (Fig. 2; $F_{3,40}=7.881, P<0.001$ ). Confluent monolayers treated to the NO donor SNAP showed no difference in permeability at either 6 or $24 \mathrm{~h}$ of exposure when compared with control. In contrast, BBMECs exposed to the longer-acting NO donor DETA-NONOate showed a significant increase in permeability at $24 \mathrm{~h}$ with both concentrations (10 
Table 1. Total nitric oxide levels from BBMECs

\begin{tabular}{lll}
\hline \hline \multicolumn{1}{c}{ Treatment } & $\begin{array}{c}\text { Control } \\
\text { (Culture medium) }\end{array}$ & $\begin{array}{r}\text { L-NAME Treated } \\
\text { (Culture medium) }\end{array}$ \\
\hline Normoxic (control) & $126.9 \pm 20.7$ & $235.5 \pm 40.2 \dagger$ \\
Hypoxic & $400.9 \pm 95.1 *$ & $207.1 \pm 59.3 \ddagger$ \\
Posthypoxic reoxygenation & $172.3 \pm 55.2$ & $199.9 \pm 38.7$ \\
\hline
\end{tabular}

Data represent means $\pm \mathrm{SE}$ (in nmol/mg protein); $n=9$. BBMECs, bovine brain microvessel endothelial cells; L-NAME, $N^{\omega}$-nitro-L-arginine methyl ester. Data analysis was done using two-way ANOVA and Tukey's post hoc comparison: ${ }^{*} P<0.01$ compared with normoxic control; $\dagger P<0.05$ and $\ddagger P<$ 0.001 compared with respective control. Nitric oxide in culture medium without cells was $<20 \%$ of that in culture medium from BBMECs independent of treatment.

and $25 \mu \mathrm{M})$, whereas only the higher concentration $(25 \mu \mathrm{M})$ had an effect at 6 h (Fig. 2).

Western blot analysis of NOS proteins. Expression of iNOS and eNOS isoforms was examined after exposure to normoxic $(\mathrm{CN} ; 24 \mathrm{~h})$, hypoxic $(\mathrm{CH} ; 24 \mathrm{~h})$, or posthypoxic reoxygenation (CHR; $2 \mathrm{~h}$ ) conditions and after pretreatment with L-NAME (LN, LH, and LHR, respectively). Western blot analysis of eNOS protein expression showed no difference in BBMECs exposed to hypoxia or posthypoxic reoxygenation compared with the normoxic group (Fig. 3A). In contrast, a significant increase $(P<0.05)$ in iNOS protein expression was observed in hypoxia-treated BBMECs compared with normoxic controls (Fig. 3B). Dissimilar from control BBMECs (culture medium alone), those pretreated with L-NAME before exposure to

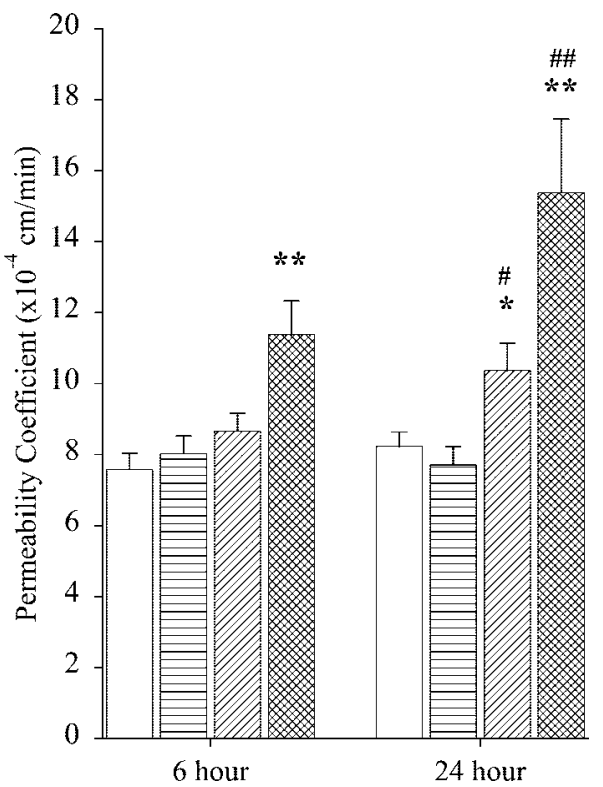

Fig. 2. Permeability coefficient of BBMECs treated with the NO donors $S$-nitroso- $N$-acetyl-DL-penicillamine (SNAP), and diethylenetriaamine NONOate (DETA-NONOate) and exposed to normoxia (6 or $24 \mathrm{~h}$ ). Open bars, monolayers in culture medium alone (control); striped bars, monolayers in culture medium with SNAP $(30 \mu \mathrm{M})$; hatched bars, monolayers treated with DETA-NONOate $(10 \mu \mathrm{M})$; crosshatched bars, monolayers treated with DETA-NONOate $(25 \mu \mathrm{M})$. Results are means \pm SE with $n=6$. Data were analyzed using two-way ANOVA with Tukey's post hoc comparison: $* P<$ $0.05, * * P<0.01$ compared with control; $\# P<0.05$, \#\#P $<0.01$ compared with 6 h treatment. $F_{3,47}=61.65$ and $P<0.001$ for differences based on changes in NO donor, $F_{1,47}=20.85$ and $P<0.001$ for differences based on exposure time and $F_{3,40}=7.88$ and $P<0.001$ for interaction between NO donor and time.
$\mathbf{A}$
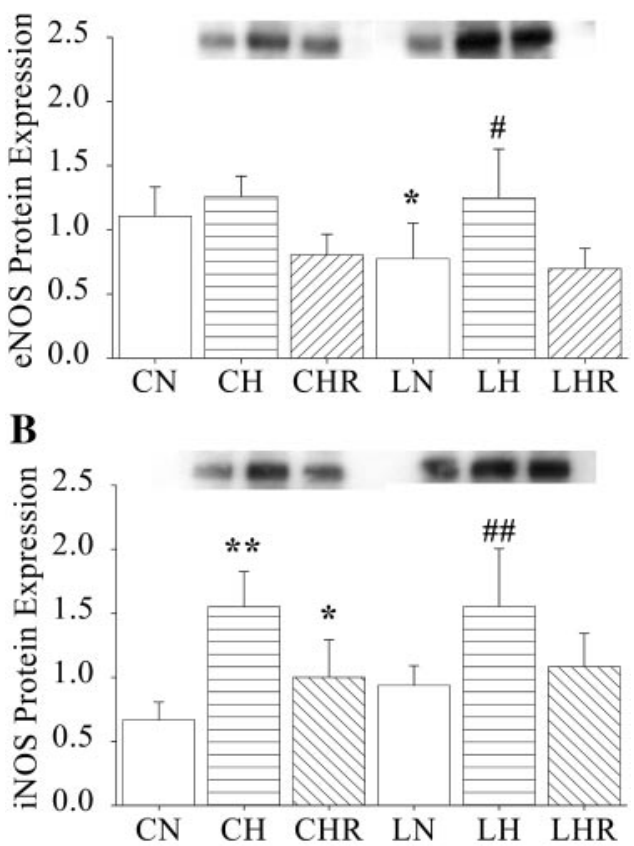

Fig. 3. Western blot analysis of NOS isoforms [endothelial NOS (eNOS) and iNOS] in BBMECs after hypoxic exposure. Confluent BBMEC monolayers were exposed to normoxic $(24 \mathrm{~h}, \mathrm{CN})$, hypoxic $(24 \mathrm{~h}, \mathrm{CH})$, or posthypoxic reoxygenation $(2 \mathrm{~h}, \mathrm{CHR})$ conditions. Similarly, monolayers were treated with L-NAME (100 nM) under similar conditions (LN, LH, and LHR, respectively). Western blot analyses for expression of eNOS (130 kDa; $A$ ) and iNOS (135 $\mathrm{kDa} ; B$ ) were performed on protein from cell lysates following treatment. Protein expression of the isoforms was normalized to the amount of protein loaded. Inset, representative Western blots with associated histograms for semiquantitative analysis of protein changes $(n=9)$. Data analyses were done with two-way ANOVA and Tukey's post hoc comparison for each isoform: $* P<0.05$ and $* * P<0.01$ compared with normoxic controls; $\# P<0.05$ and $\# \# P<0.001$ compared with respective oxygen controls.

normoxia, hypoxia, and posthypoxic reoxygenation showed changes in expression of both eNOS and iNOS. Most notable is the increase in basal level expression of iNOS on treatment with L-NAME under normoxic conditions (Fig. 3B).

\section{DISCUSSION}

Using primary BBMECs as an in vitro BBB model, we examined whether the NO pathway was involved in permeability changes seen after hypoxia and posthypoxic reoxygenation. This study describes three major findings. First, BBMECs exposed to hypoxic conditions demonstrated an increased paracellular permeability that was significantly attenuated by either reoxygenation or inhibition of NOS. To our knowledge, this is the first report where NOS inhibition in combination with posthypoxic reoxygenation resulted in complete attenuation of hypoxia-induced permeability changes in brain microvessel endothelial cells. Second, the hypoxia-induced increase in permeability paralleled a significant increase in total NO levels. Finally, changes in permeability and total NO levels after exposure to hypoxic conditions were linked to similar changes in total protein expression of iNOS with little change in eNOS.

Similar to our previous observations (30), Fig. 1 shows a 2.3 -fold increase in $\left[{ }^{14} \mathrm{C}\right]$ sucrose permeability of BBMEC 
monolayers exposed to hypoxia compared with normoxic controls and a reduction $(51 \%)$ in this effect with posthypoxic reoxygenation. The present study expands on this finding by demonstrating that pretreatment of BBMEC monolayers with either a nonselective NOS inhibitor (L-NAME, $100 \mathrm{nM}$ ) or iNOS-selective inhibitor (1400W, $100 \mathrm{nM}$ ) also had a significant attenuating effect on hypoxia-induced increased permeability (Fig. 1). Moreover, the combination of L-NAME or $1400 \mathrm{~W}$ with posthypoxic reoxygenation improved the attenuating effect on hypoxia-induced permeability changes. In fact, inhibition of $\mathrm{NO}$ in combination with reoxygenation resulted in a permeability coefficient similar to normoxic controls (6.52 or 6.17 compared with $\left.4.79 \mathrm{~cm} / \mathrm{min} \times 10^{-4}\right)$. Some studies (13, $17,27,47)$ have demonstrated a release of $\mathrm{NO}$ as a result of hypoxic stress, whereas others $(22,31,45)$ have examined the release of NO during reperfusion or reoxygenation. Few (13) have reported a beneficial response to hypoxic stress by inhibition of NOS in cerebral microvessel endothelial cells, and, to our knowledge, this is the first report of permeability changes in cerebral microvessel endothelial cells related to NO during hypoxia and/or posthypoxic reoxygenation within the same experimental paradigm. The attenuation of hypoxia-induced paracellular permeability changes by inhibition of NO synthesis alone or in combination with a return to normoxic conditions is critical in understanding cerebral microvascular cell dysfunction that occurs during hypoxic stress and/or posthypoxic reoxygenation. Whereas these data suggest that NO is a significant contributor to increased permeability during hypoxic stress, further investigation is necessary to understand what other cellular mediators (i.e., cytokines or ROS) and/or mechanisms are activated by hypoxic stress, which are attenuated by reoxygenation rather than inhibition of NOS.

In conjunction with changes in paracellular permeability, a significant increase (3.1-fold) in total NO level was observed in the culture medium of BBMEC monolayers exposed to hypoxia compared with normoxic controls (Table 1). The concentration of $\mathrm{NO}$ and its metabolites decreased by $86 \%$ on posthypoxic reoxygenation compared with the hypoxia-treated BBMECs. This decrease in total NO concentration parallels the changes seen in paracellular permeability (i.e., attenuation of the hypoxia-induced hyperpermeability effect on reoxygenation; Fig. 1). Furthermore, L-NAME treatment resulted in a decreased concentration of $\mathrm{NO}$ and its metabolites during hypoxic conditions compared with hypoxia without L-NAME treatment (Table 1).

NO has been shown to have beneficial effects as a vasodilatory agent $(31,49)$ as well as have detrimental effects such as increased permeability in the mesenteric and cerebral microvasculature $(4,32)$. Whereas NO has a wide range in half-life due to alterations in synthesis and degradation, these processes are dependent on the concentration of $\mathrm{NO}$ as well as the molecular environment of the tissue (see Refs. 19 and 24 for reviews). This observation may explain the increased total NO during hypoxic conditions and its rapid decline or metabolism on reoxygenation. Perhaps the metabolic pathway of NO or breakdown of its metabolites (i.e., $\mathrm{NO}_{2}^{-}$and $\mathrm{NO}_{3}^{-}$), which accumulate during the hypoxic insult, is altered with the rapid increase in $\mathrm{PO}_{2}$ during reoxygenation.

The primary degradation products of $\mathrm{NO}$ are $\mathrm{NO}_{2}^{-}$and $\mathrm{NO}_{3}^{-}$, but NO can also interact with heme proteins, ROS such as superoxide anion and hydrogen peroxide, and with itself to nitrosate or nitrosylate amino acid residues. It has been suggested that the reaction of NO with these oxygen radicals and proteins may be important in the breakdown of $\mathrm{NO}$ and be dependent on the concentration of these substances in various cells or tissues. Mason et al. (31) reported a protective effect of NO by demonstrating increased NO release concomitant with a reduced level of ROS in passaged cerebral capillary endothelial cells afer postanoxic reoxygenation. Whereas the treatment conditions were different from those used in our study, these results suggest that alterations in the level of $\mathrm{NO}$ and its metabolites during a hypoxic and posthypoxic reoxygenation event may depend on a decrease in NO synthesis or increase in metabolism and/or degradation.

Because NO is a gaseous molecule with a very short lifespan that makes it difficult to accurately measure or assess its effect, we used two different time points: two NO donors of varying half-life and two concentrations of one donor to assess the effects of $\mathrm{NO}$ on permeability in BBMECs. Increased $\left[{ }^{14} \mathrm{C}\right]$ sucrose permeability in BBMEC monolayers has been seen as early as $6 \mathrm{~h}$ hypoxia with a maximal effect at $24 \mathrm{~h}$ (data not shown), which outlined the time points examined with the NO donors in this study. Furthermore, we used NO donors with different half-lives and different concentrations, which would release NO at or near levels previously used in other cellular systems $(2,29,39)$. The idea that hypoxia-induced hyperpermeability via NO is supported by our observations where NO released from DETANONOate had a direct effect on paracellular permeability in BBMECs (Fig. 2). These data also suggest that exposure time and concentration of NO may be determinants in the severity of brain microvessel endothelium dysfunction during hypoxia because the higher concentration and longer half-life NO-donor had the most significant effect on permeability.

NO donors, such as sodium nitroprusside and 3-morpholinosydnonimine, have been shown to potentiate rather than inhibit cytoskeletal contraction in a cGMP-independent manner (7). Furthermore, this action on the cytoskeleton was blocked by inhibition of PKC. These NO donors also have been shown to increase hepatocyte permeability, a known effect of PKC in this cell type, indicating that exogenous NO modulates the apicolateral cytoskeleton of hepatocytes via PKC activation rather than via cGMP or intracellular calcium (7). Ogawa et al. (36) demonstrated decreased levels of cAMP and adenyl cyclase activity, which correlated to increased permeability in endothelial monolayers exposed to hypoxic conditions. This effect was inhibited by the addition of dexamethasone (potentially by inhibition of phospholipase or PKA). Abbruscato et al. (1) showed that calcium channel inhibitors (nifedipine and SK/F-96365) reduced hypoxia \& aglycemia-induced increases in BBMEC permeability. Because NO is produced constitutively by calcium-dependent eNOS and by calcium-independent iNOS, alterations in calcium levels may impact NO formation and other calciumdependent cellular mechanisms (e.g., kinase activation). Whereas NO is a membrane-permeable mediator that can modulate soluble guanylate cyclase, phosphokinases (PKA and PKG), phosphodiesterases, and transcription factors or react with amino acid residues (i.e., nitrosated or nitrosylated proteins) $(7,8,12,19,35)$, it is currently unknown whether these second messenger pathways are modulated by $\mathrm{NO}$ in the 
cerebral microvasculature following hypoxic stress or posthypoxic reoxygenation.

It has been suggested that inflammatory mediators are also important factors in contributing to ischemic brain injury (10, 44). An increase in VEGF has been correlated with increased cerebral microvascular permeability in hypoxia-treated mice (42), and this effect has been linked to activation of NO release and the subsequent increase in permeability in vitro $(13,14)$. Separate from the NO-signaling pathway, hypoxia-induced cytokines may activate other intracellular pathways, which contribute to the total increase in permeability seen in the present study. In addition to VEGF, hypoxia has been associated with an increased release of other cytokines from the microvasculature (3), where an increased release of interleukin-6 was correlated with decreased transendothelial resistance, i.e., increased permeability in HUVEC cells exposed to hypoxia $\left(1.5 \% \mathrm{O}_{2}\right)$. Examination of other intracellular signaling pathways (e.g., prostaglandins or kinases) that are stimulated by these cytokines may clarify the NO-independent mechanisms that contribute to hypoxia-induced hyperpermeability under the current experimental paradigm.

Selective inhibition of the iNOS isoform has been demonstrated both in vivo and in vitro $(15,34)$, and the use of $1400 \mathrm{~W}$ has been shown to reduce cerebral infarct size in vivo $(21,38)$. This supports the present study where a significant increase in iNOS expression (Fig. 3), total NO production (Table 1), and increased paracellular permeability (Fig. 1) was seen with hypoxic-treated BBMECs. Both the nonselective NOS inhibitor (L-NAME) and selective iNOS inhibitor (1400W) had similar attenuating effects in paracellular permeability during both hypoxia and posthypoxic reoxygenation treatments. This supports our hypothesis that a hypoxic insult stimulates NO production via the inducible isoform iNOS ultimately resulting in increased permeability of the cerebral microvasculature. Whereas there was no significant difference in the expression of total eNOS among the treatment groups (Fig. 3), the possibility remains that activation of this isoform may occur under this experimental paradigm. Although it is unknown whether eNOS and iNOS may be differentially regulated during hypoxic and posthypoxic stress, measuring specific NOS isoform activity under these conditions will improve our understanding of how this cellular mediator is regulated. It is interesting to note that treatment with L-NAME alone caused an increase in iNOS expression, which may explain the increase in basal levels of NO released in L-NAME-treated groups (Table 1 and Fig. 3).

Stimulation of different NOS isoforms (i.e., eNOS and iNOS) has been shown to have different effects on paracellular permeability in human umbilical vein endothelial cell monolayers (8). Whereas nanomolar levels of estradiol stimulated eNOS, resulting in decreased paracellular permeability, micromolar concentrations stimulated iNOS, resulting in increased permeability (8). The change in iNOS expression and increased release of NO in the cerebral microvessel endothelial cells may involve increased expression of hypoxia-inducible factor- $1 \alpha$, which has been shown to activate transcription of iNOS gene expression in the pulmonary and cardiac endothelium following hypoxic exposure $(23,37)$. Data from the present study and others $(8,16)$ suggest that eNOS and iNOS activity or protein expression may be regulated by the degree of stimulus or stress and that the total NO level may differentially modulate endo- thelial cell monolayer integrity. This is supported by our findings where increased total NO levels and iNOS expression parallel the increased paracellular permeability after hypoxic stress and that these effects are reduced on reoxygenation (Table 1 and Fig. 3). Whereas a potential contribution by eNOS activity needs further clarification and the effects of L-NAME on iNOS and eNOS expression and the basal levels of NO remain unclear, it is possible that, due to location, NO produced by eNOS maintains barrier integrity, and NO produced by iNOS activates a signaling pathway(s) that leads to barrier dysfunction.

In summary, a hypoxic insult stimulates increased iNOS expression and total NO production, which correlate with increased paracellular permeability. This disruption in cerebral microvascular integrity is attenuated by inhibition of NOS. Whereas NO formation appears to contribute to hypoxiainduced increased paracellular permeability, other cellular mediators and/or mechanisms (i.e., calcium modulation, cytokines, nitrosylation or phosphorylation of proteins, or PKC activation) that may be involved in disrupting tight junctional protein complexes require additional investigation. Understanding the sequence of cellular events stimulated by hypoxic stress and posthypoxic reoxygenation within cerebral microvascular endothelial cells will provide insight to develop better preventive measures and therapeutic approaches to reduce neuronal tissue damage that occurs following ischemic stroke and/or high-altitude exposure.

\section{GRANTS}

This study was supported by National Institutes of Health Grants R01-NS39592 and F32-NS-11175-01.

\section{REFERENCES}

1. Abbruscato TJ and Davis TP. Combination of hypoxia/aglycemia compromises in vitro blood-brain barrier integrity. J Pharmacol Exp Ther 289: 668-675, 1999.

2. Adachi R, Matsui S, Kinoshita M, Nagaishi K, Sasaki H, Kasahara T, and Suzuki K. Nitric oxide induces chemotaxis of neutrophil-like HL-60 cells and translocation of cofilin to plasma membranes. Int J Immunopharmacol 22: 855-864, 2000.

3. Ali MH, Schlidt SA, Chandel NS, Hynes KL, Schumacker PT, and Gewertz BL. Endothelial permeability and IL-6 production during hypoxia: role of ROS in signal transduction. Am J Physiol Lung Cell Mol Physiol 277: L1057-L1065, 1999.

4. Baldwin AL, Thurston G, and al Naemi H. Inhibition of nitric oxide synthesis increases venular permeability and alters endothelial actin cytoskeleton. Am J Physiol Heart Circ Physiol 274: H1776-H1784, 1998.

5. Betz AL, Yang GY, Kawai N, Fujisawa M, Abdelkarim GE, Ennis SR, and Keep RF. Reperfusion-induced injury of the blood-brain barrer. In: Brain Barrier Systems, edited by Paulson OB, Knudsen GM, and Moos T. Copenhagen, Denmark: Munksgaard, 1999, p. 430-440.

6. Bolanos JP and Almeida A. Roles of nitric oxide in brain hypoxia-ischemia. Biochim Biophys Acta 1411: 415-436, 1999.

7. Burgstahler AD and Nathanson MH. NO modulates the apicolateral cytoskeleton of isolated hepatocytes by a PKC-dependent, cGMP-independent mechanism. Am J Physiol Gastrointest Liver Physiol 269: G789G799, 1995.

8. Cho MM, Ziats NP, Pal D, Utian WH, and Gorodeski GI. Estrogen modulates paracellular permeability of human endothelial cells by eNOSand iNOS-related mechanisms. Am J Physiol Cell Physiol 276: C337C349, 1999.

9. Chryssanthou C, Palaia T, Goldstein G, and Stenger R. Increase in blood-brain barrier permeability by altitude decompression. Aviat Space Environ Med 58: 1082-1086, 1987.

10. Del Zoppo G, Ginis I, Hallenbeck JM, Iadecola C, Wang X, and Feuerstein GZ. Inflammation and stroke: putative role for cytokines, 
adhesion molecules and iNOS in brain response to ischemia. Brain Pathol 10: 95-112, 2000.

11. Deli MA, Descamps L, Dehouck MP, Cecchelli R, Joo F, Abraham CS, and Torpier G. Exposure of tumor necrosis factor- $\alpha$ to luminal membrane of bovine brain capillary endothelial cells co-cultured with astrocytes induces a delayed increase of permeability and cytoplasmic stress fiber formation of actin. J Neurosci Res 41: 717-726, 1995.

12. Demiryurek AT, Karamsetty MR, McPhaden AR, Wadsworth RM, Kane KA, and MacLean MR. Accumulation of nitrotyrosine correlates with endothelial NO synthase in pulmonary resistance arteries during chronic hypoxia in the rat. Pulm Pharmacol Ther 13: 157-165, 2000.

13. Fischer S, Clauss M, Wiesnet M, Renz D, Schaper W, and Karliczek GF. Hypoxia induces permeability in brain microvessel endothelial cells via VEGF and NO. Am J Physiol Cell Physiol 276: C812-C820, 1999.

14. Fischer S, Wobben M, Marti HH, Renz D, and Schaper W. Hypoxiainduced hyperpermeability in brain microvessel endothelial cells involves VEGF-mediated changes in the expression of zonula occludens-1. Microvasc Res 63: 70-80, 2002.

15. Garvey EP, Oplinger JA, Furfine ES, Kiff RJ, Laszlo F, Whittle BJ, and Knowles RG. $1400 \mathrm{~W}$ is a slow, tight binding, and highly selective inhibitor of inducible nitric-oxide synthase in vitro and in vivo. $J$ Biol Chem 272: 4959-4963, 1997.

16. Gonzalez NC and Wood JG. Leukocyte-endothelial interactions in environmental hypoxia. Adv Exp Med Biol 502: 39-60, 2001.

17. Guo Y, Ward ME, Beasjours S, Mori M, and Hussain SN. Regulation of cerebellar nitric oxide production in response to prolonged in vivo hypoxia. J Neurosci Res 49: 89-97, 1997.

18. Hackett PH. High altitude cerebral edema and acute mountain sickness. A pathophysiology update. Adv Exp Med Biol 474: 23-45, 1999.

19. Hanafy KA, Krumenacker JS, and Murad F. NO, nitrotyrosine, and cyclic GMP in signal transduction. Med Sci Monit 7: 801-819, 2001.

20. Huang PL. Mouse models of nitric oxide synthase deficiency. J Am Soc Nephrol 11, Suppl 16: S120-S123, 2000.

21. Iadecola $\mathbf{C}$, Zhang $\mathbf{F}$, and $\mathbf{X u} \mathbf{X}$. Inhibition of inducible nitric oxide synthase ameliorates cerebral ischemic damage. Am J Physiol Regul Integr Comp Physiol 268: R286-R292, 1995.

22. Ioroi T, Yonetani M, and Nakamura H. Effects of hypoxia and reoxygenation on nitric oxide production and cerebral blood flow in developing rat striatum. Pediatr Res 43: 733-737, 1998.

23. Jung F, Palmer LA, Zhou N, and Johns RA. Hypoxic regulation of inducible nitric oxide synthase via hypoxia inducible factor- 1 in cardiac myocytes. Circ Res 86: 319-325, 2000.

24. Kelm M. Nitric oxide metabolism and breakdown. Biochim Biophys Acta 1411: 273-289, 1999.

25. Kish PE and Ueda T. Calcium-dependent release of accumulated glutamate from synaptic vesicles within permeabilized nerve terminals. Neurosci Lett 122: 179-182, 1991.

26. Kristian T and Siesjo BK. Calcium in ischemic cell death. Stroke 29: 705-718, 1998.

27. Liang M and Knox FG. Production and functional roles of nitric oxide in the proximal tubule. Am J Physiol Regul Integr Comp Physiol 278: R1117-R1124, 2000.

28. Lum H, Barr DA, Shaffer JR, Gordon RJ, Ezrin AM, and Malik AB. Reoxygenation of endothelial cells increases permeability by oxidantdependent mechanisms. Circ Res 70: 991-998, 1992.

29. Maccarrone M, Putti S, and Finazzi Agro A. Nitric oxide donors activate the cyclo-oxygenase and peroxidase activities of prostaglandin $\mathrm{H}$ synthase. FEBS Lett 410: 470-476, 1997.

30. Mark KS and Davis TP. Cerebral microvascular changes in permeability and tight junctions induced by hypoxia-reoxygenation. Am J Physiol Heart Circ Physiol 282: H1485-H1494, 2002.
31. Mason RB, Pluta RM, Walbridge S, Wink DA, Oldfield EH, and Boock RJ. Production of reactive oxygen species after reperfusion in vitro and in vivo: protective effect of nitric oxide. J Neurosurg 93: 99-107, 2000.

32. Mayhan WG. Role of nitric oxide in disruption of the blood-brain barrier during acute hypertension. Brain Res 686: 99-103, 1995.

33. Miller DW, Audus KL, and Borchardt RT. Application of cultured endothelial cells of the brain microvasculature in the study of the bloodbrain barrier. J Tiss Cult Meth 14: 217-224, 1992.

34. Murray IA, Daniels I, Coupland K, Smith JA, and Long RG. Increased activity and expression of iNOS in human duodenal enterocytes from patients with celiac disease. Am J Physiol Gastrointest Liver Physiol 283: G319-G326, 2002.

35. Nobles M and Abbott NJ. Modulation of the effects of extracellular ATP on $\left[\mathrm{Ca}^{2+}\right]_{\mathrm{i}}$ in rat brain microvacular endothelial cells. Eur J Pharmacol 361: 119-127, 1998.

36. Ogawa S, Koga S, Kuwabara K, Brett J, Morrow B, Morris SA, Bilezikian JP, Silverstein SC, and Stern D. Hypoxia-induced increased permeability of endothelial monolayers occurs through lowering of cellular cAMP levels. Am J Physiol Cell Physiol 262: C546-C554, 1992.

37. Palmer LA, Semenza GL, Stoler MH, and Johns RA. Hypoxia induces type II NOS gene expression in pulmonary artery endothelial cells via HIF-1. Am J Physiol Lung Cell Mol Physiol 274: L212-L219, 1998.

38. Parmentier S, Bohme GA, Lerouet D, Damour D, Stutzmann JM, Margaill I, and Plotkine M. Selective inhibition of inducible nitric oxide synthase prevents ischaemic brain injury. Br J Pharmacol 127: 546-552, 1999.

39. Raychaudhuri B, Dweik R, Connors MJ, Buhrow L, Malur A, Drazba J, Arroliga AC, Erzurum SC, Kavuru MS, and Thomassen MJ. Nitric oxide blocks nuclear factor- $\beta$ activation in alveolar macrophages. Am J Respir Cell Mol Biol 21: 311-316, 1999.

40. Rosenberg GA. Ischemic brain edema. Prog Cardiovasc Dis 42: 209216, 1999.

41. Samdani AF, Dawson TM, and Dawson VL. Nitric oxide synthase in models of focal ischemia. Stroke 28: 1283-1288, 1997.

42. Schoch HJ, Fischer S, and Marti HH. Hypoxia-induced vascular endothelial growth factor expression causes vascular leakage in the brain. Brain 125: 2549-2557, 2002.

43. Shinnou M, Ueno M, Sakamoto H, and Ide M. Blood-brain barrier damage in reperfusion following ischemia in the hippocampus of the mongolian gerbil. Acta Neurol Scand 98: 406-411, 1998.

44. Stanimirovic D and Satoh K. Inflammatory mediators of cerebral endothelium: a role in ischemic brain inflammation. Brain Pathol 10: 113-126, 2000.

45. Utepbergenov DI, Mertsch K, Sporbert A, Tenz K, Paul M, Haseloff RF, and Blasig IE. Nitric oxide protects blood-brain barrier in vitro from hypoxia/reoxygenation-mediated injury. FEBS Lett 424: 197-201, 1998.

46. Xu J, He L, Ahmed SH, Chen SW, Goldberg MP, Beckman JS, and Hsu CY. Oxygen-glucose deprivation induces inducible nitric oxide synthase and nitrotyrosine expression in cerebral endothelial cells. Stroke 31: 1744-1751, 2000.

47. Xu XP, Pollock JS, Tanner MA, and Myers PR. Hypoxia activates nitric oxide synthase and stimulates nitric oxide production in porcine coronary resistance arteriolar endothelial cells. Cardiovasc Res 30: 841847, 1995.

48. Yang GY and Betz AL. Reperfusion-induced injury to the blood-brain barrier after middle cerebral artery occlusion in rats. Stroke 25: 1658 1665, 1994.

49. Zech JC, Pouvreau I, Cotinet A, Goureau O, Le Varlet B, and de Kozak Y. Effect of cytokines and nitric oxide on tight junctions in cultured rat retinal pigment epithelium. Invest Ophthalmol Vis Sci 39: 1600-1608, 1998. 